\title{
The mental health care act 2017: A critical review
}

\author{
Sireesha Srinivas Rao \\ Professor, Institute of Mental Health, Hyderabad, Telangana, India \\ *Corresponding Author: Sireesha Srinivas Rao \\ Email: s283572@gmail.com
}

Mental health care act 2017 (MHCA 2017) received assent from the President of India on April 2017 and came into force on 7th April 2018. The act was described as an act to provide mental health care and services to protect, promote and fulfill the rights of such persons during delivery of mental health care and services and for matters connected there with or incidental there to. It superseded the previously existing mental health act 1987 (MHA 1987).

I have been working in the government institute for mental health care for over a decade, in the era of MHA 87. Working now with the new mental health care act in the government sector has its share of difficulties. Though there are a lot of grey areas in the act, it is not without its positives. However in the current review I would be highlighting the grey areas which would be challenging in day to day practice.

\section{Definition of Mental Illness}

Chapter 1 of the MHCA 2017 defines mental illness a substantial disorder of thinking, mood, perception, orientation or memory that grossly impairs judgement, behavior, capacity to recognize reality or ability to meet the ordinary demands of life, mental conditions associated with the abuse of alcohol and drugs, but does not include mental retardation which is a condition of arrested or incomplete development of mind of a person, specially characterized by sub normality of intelligence. This definition in my view is too restrictive. Going entirely by this definition, disorders like conversion disorder, phobia, panic disorders and personality disorders which are mental illnesses as per international classification of diseases (ICD 10), get excluded. Further adding to the confusion is the section 2 of MHCA 2017 which states that the determination of mental illness is as per national or international guidelines like ICD or Diagnostic and statistical manual (DSM).So there is a dilemma whether to follow the definition of MHCA 2017 or ICD 10.Hence a grey area.

\section{Admission Procedure}

Sometimes it is not possible to make a diagnosis of a disorder in a single interview. Admission into a mental health establishment (MHE) may be needed for observation and serial mental status evaluation. However such a provision is not available in the new act. Admissions in to MHE is possible only under sections $86,88,89$ or 94 .Sometimes admission and treatment may be needed to stop the progression to severe illness which is not possible under the new act. We are forced to wait till condition of the person with mental illness (PWMI) becomes severe as per MHCA 2017 and then consider admitting him.
Let's take another instance of a PWMI being diagnosed as severe depression with suicidal ideation admitted under sec. 89 of the MHCA.She requires urgent electroconvulsive therapy (ECT) in the opinion of the treating psychiatrist. However in her advance directive the PWMI specifically mentions no ECT and no antidepressants. The psychiatrist has to then write to the board and wait for overriding the $\mathrm{AD}$ thereby loosing precious time, as many times ECT is a lifesaving procedure for suicidal patients. In my opinion it has to be purely the treating psychiatrist's clinical judgement whether or not to give ECT.

Even when admission is involuntary, Psychiatrists need to take into account advance directive (AD)/ consent of nominated representative (NR)/ assess capacity periodically and treat. This is time consuming, resource consuming and a hindrance in patient care and discharge of duties by the psychiatrist. My contention is when the PWMI is admitted as per section 89 , there is no need to look in to advance directive again.

The board has a say in admissions, which becomes mandatory for admission as per section 90 and also in modality of treatment and consent. However, the discharge planning has to be done by the psychiatrist. In my opinion it is an unfair burden on the psychiatrist who has no say in the admission but is made responsible for discharge.

\section{Assessment of Capacity}

As per section 81 of MHCA 2017, the central authority shall appoint an expert committee to prepare a guidance document for assessment of capacity. Such an authority has not yet been formed in most places. Till such a time, psychiatrists are in dilemma as to how to assess capacity. There are also other grey areas regarding capacity. For instance, if the PWMI has capacity but does not want treatment then the mental health professional (MHP) cannot treat him, even if he needs treatment (even after admission as per section 89).

If PWMI does not have capacity and if the NR is not available then the psychiatrist cannot extend any help, till NR is appointed by the board. All this will lead to loss of manpower, time and lot of paperwork, further adding to woes of the already scarce mental health resources.

\section{Advance Directive}

It is difficult to determine whether the PWMI at the time of making the AD had the capacity to do so, as it was made by him in the past. It is also difficult to determine whether he was certified by a psychiatrist at the time of making the AD. Even if he did have capacity to make the $A D$, given the 
educational, socioeconomic and cultural background of our patients how many are capable of making correct health care decisions and how many will conform to standard guidelines of treatment is debatable.

AD can be revoked, amended or cancelled by the person who has made it any number of times. This will put a lot of burden and not practical to implement.

\section{Nominated Representative}

One can never be sure of the intentions, education and the understanding of the NR about mental illness and their capacity to act in best interests of patients.

In our culture responsibility of caregiving of the PWMI is taken up by the entire family and in such a scenario giving responsibility of taking decisions of care to a single person puts a lot of burden on the NR and may also disrupt family relations in some cases.

PWMI can be admitted to MHE against his or her wish only upon application from the NR. Such a decision against PWMI by the NR may lead to anger and hatred towards the NR.

If NR is not acting in the best interests of PWMI, relative of PWMI / MHP taking care of person can apply to the board for revocation of NR.Psychiatrist cannot take decision independently without approval from board even if it is in the best interests of the PWMI.

In case of PWMIs who have been staying in the hospital for long periods, when they regain the capacity to take decisions they can request for discharge. Family members are often not willing to accept them. I come across such scenarios regularly in the hospital in which I work in. Psychiatrists are caught up in the dilemma of whether to follow MHCA 2017 and discharge the PWMI or to keep them in the hospital.

\section{Rights of PWMI}

Section 20 says not to subject to compulsory tonsuring. But we all know that PWMI are sometimes found in a disheveled state, wandering on the roads and don't have the capacity to give consent or make decisions for themselves and neither do they have an $\mathrm{AD} / \mathrm{NR}$.It is practically impossible to manage such patients.

Section 20 also talks of their right to wear personal clothes if so wished and to not force them to wear uniform. In my view PWMI wearing uniforms serve many purposes like uniformity, easy identification and safety from suicidal attempts.

\section{Registration of Mental Health Establishment}

As per section 65, MHCA 2017, before issuing provisional registration to $\mathrm{MHE}$, authority won't be inspecting MHE physically. Whereas under MHA 87, licenses were issued only after inspecting the MHE.Though it expedites the process, glaring lacunae are overlooked.

\section{Mental Health Review Boards}

Section 78 says proceedings before board are to be judicial proceedings - which means Psychiatrists who are having mental health establishment under their name should hire / appoint a lawyer, which is a costly affair.

Section 95 clubs ECT for minors along with prohibited procedures like sterilization of men or women and requires prior permission of the review board. This in my opinion is not fair as ECT is a lifesaving procedure and further worsens stigma towards it.

\section{Offences and Penalties}

Section 107, MHCA 2017 says penalties for establishing or maintaining MHE in contravention of provisions of this act like running a MHE without registration shall be liable for punishment up to Rs 5000.

In such a scenario if a PWMI has problems which require services of a psychiatrist and a general physician in a general hospital, such setting should have been registered as per MHCA 2017.If it is not the case, psychiatrist can offer services for only 72 hours (section 74). After 72 hours if he needs services of a psychiatrist, either he has to be shifted to a MHE or a registered corporate hospital as per the new act. Psychiatrists are in dilemma whether to treat such PWMI or check for registration or shift to mental health establishment. Such challenges come to fore while dealing with cases such as delirium. After MHCA comes into force hospitals may refuse admissions if there is any history of psychiatric illness. In my opinion it will further increase stigma towards mental illness.

The act is not without positives. However the grey areas have to be addressed to make it more effective.

\section{Acknowledgment}

Dr. Srilakshmi, Associate Professor, Gandhi medical college, Secunderabad for encouragement and support.

\section{Conflict of Interest: None.}

\section{References}

1. Mental health care act 2017. 